\title{
Philosophiques
}

\section{Yves Charles Zarka, Un détail nazi dans la pensée de Carl Schmitt, Paris, Presses Universitaires de France, Collection Intervention philosophique, 2005, 96 pages.}

\section{Luc Vigneault}

Volume 34, numéro 1, printemps 2007

Cosmopolitisme et particularisme

URI : https://id.erudit.org/iderudit/015879ar

DOI : https://doi.org/10.7202/015879ar

Aller au sommaire du numéro

Éditeur(s)

Société de philosophie du Québec

ISSN

0316-2923 (imprimé)

1492-1391 (numérique)

Découvrir la revue

Citer ce compte rendu

Vigneault, L. (2007). Compte rendu de [Yves Charles Zarka, Un détail nazi dans la pensée de Carl Schmitt, Paris, Presses Universitaires de France, Collection Intervention philosophique, 2005, 96 pages.] Philosophiques, 34(1), 220-223. https://doi.org/10.7202/015879ar d'utilisation que vous pouvez consulter en ligne.

https://apropos.erudit.org/fr/usagers/politique-dutilisation/ 


\section{Yves Charles Zarka, Un détail nazi dans la pensée de Carl Schmitt, Paris, Presses Universitaires de France, Collection Intervention philosophique, 2005, 96 pages.}

La polémique entourant la collaboration de certains intellectuels allemands avec le régime nazi n'a rien de nouveau. Le cas de Heidegger a encore récemment été passé à la loupe par Emmanuel Faye, et le débat a été très vif. Il n'est donc pas étonnant que les écrits de Carl Schmitt apparaissent au banc des accusés. Quiconque connaît un tant soit peu le juriste et philosophe Carl Schmitt ne contestera non seulement son adhésion au parti national-socialiste allemand (NSDAP) ni ses prises de position et son engagement en faveur de l'idéologie hitlérienne mais aussi, et surtout, le fait que jusqu'à sa mort il n'exprima aucune forme de regret sur sa collaboration avec le régime nazi et la légitimité de celui-ci. Nul doute, Carl Schmitt fut bel et bien un nazi consentant et conscient des actions que ce régime présupposait. La question reste maintenant de savoir si les concepts juridiques et philosophiques de celui qui se considérait lui-même comme le fils spirituel de Hobbes ont encore, malgré ces faits, une quelconque valeur théorique. Que les idées de Schmitt aient trouvé preneur du côté de l'extrême droite n'est pas une chose surprenante. La question, plus problématique en effet, est que depuis quelque temps cette appropriation n'est plus l'unique apanage des ultra-libéraux ou des néo-conservateurs, mais de plusieurs penseurs importants de l'extrême gauche. Étonnant, mais comment comprendre une telle chose ? Outre les nombreux colloques, ouvrages ou articles, récemment consacrés à la pensée de Schmitt, on trouve actuellement chez les penseurs - clés du néo-marxisme (Toni Negri, Étienne Balibar ou Giorgio Agamben, pour ne nommer que ceux-là) une réappropriation des thèses de Schmitt, réappropriation qui viendrait pallier une «crise» de la pensée marxiste. Comment un auteur comme Carl Schmitt peut-il alimenter des discours de gauche ? La « crise très profonde de la gauche postmarxiste » offrirait-elle l'occasion à la théorie schmittienne de substituer la critique bien connue de Marx tant du « libéralisme, du parlementarisme, de la représentation que de la formalité des droits de l'homme» ? (p. 92) Telle sera la question que pose Zarka à propos de cette surprenante polémique sur la pensée de Schmitt.

Le contexte dans lequel s'inscrit le petit livre de Yves Charles Zarka est une sorte d'urgence à prendre position dans cette polémique et suit une série d'articles qu'a publiés l'auteur depuis 2002 sur le sujet. La prise de position de Zarka ne prétend pas faire le tour de la question «Schmitt» — l'auteur nous annonce qu'il prépare un autre ouvrage sur Carl Schmitt - mais, au contraire, simplement prendre position dans cette réappropriation insensée. La barbarie nazie ne s'est pas mise en place sans une longue et savante préparation : loin d'être un événement accidentel, une faille dans un parcours, elle est plutôt le fruit d'une idéologie, d'un travail de la pensée qui a développé et légitimé la nécessité même de sa réalisation. Ce sont les idées comme telles, propres à ce genre d'idéologie « qui conduisent aux ténèbres que [Zarka] appelle meurtrières » (p. 10). Parmi les fabricateurs de ces idées, Carl Schmitt occupe une place privilégiée, et cela pour deux raisons : pour la réappropriation dont il est l'objet mais aussi pour la radicalité avec laquelle il mettra sa pensée au service d'une légitimation de cette barbarie.

L'essentiel de l'ouvrage de Zarka sera de cerner la principale contribution de Schmitt à l'idéologie nazie dans la définition du juif et de la race juive comme " ennemi » intérieur et extérieur du régime nazi et du peuple allemand, définition dont découlera l'exposition de la position schmittienne d'une légitimation théorique de l'élimination 
de cet «ennemi ». Si, pour Schmitt, le dualisme «ami/ennemi » est une catégorie naturelle de la chose politique - au sens où tout rapport politique doit se penser selon le fait naturel de la lutte entre antagonistes et donc de la catégorie ami/ennemi - la particularité de l'ennemi juif est que, loin d'être un ennemi ordinaire, un ennemi relationnel (un ennemi qui résulte de circonstances particulières et qui peut donc, dans une autre circonstance redevenir ami) il est un ennemi substantiel : un ennemi de sang au-delà de toute circonstance particulière. Un ennemi ontologique. Si la catégorie reste innommée par Schmitt, elle est toutefois opérante, selon Zarka, et en particulier dans les lois de Nüremberg. Dans une telle logique délirante de la guerre des races, il ne peut y avoir de répit, écrit celui-ci, « avant que l'une ou l'autre des races l'emporte et l'autre soit exterminée » (p. 10).

Le premier chapitre intitulé «La législation raciale ou la protection du sang allemand du citoyen allemand », cherche à démontrer la profondeur de l'engagement de Schmitt dans la justification des lois de Nüremberg. Ce genre de justification, de la part de Schmitt, tend «à rendre acceptable l'inacceptable et justifiable l'injustifiable, [et] appartient au genre des productions intellectuelles meurtrières » (p. 10). Moment donc particulièrement important dans l'engagement nazi de Schmitt, la légitimation de la législation raciale des lois de Nüremberg nous montre l'importance de la collaboration directe du juriste allemand et son affinité naturelle avec l'idéologie nazie. Deux textes inédits de Schmitt, traduits par Denis Trierweiler (le traducteur d'un des ouvrages les plus importants de Schmitt: Le Léviathan dans la théorie de l'État de Thomas Hobbes) que l'on retrouve dans la seconde moitié du livre, seront plus particulièrement visés ici : La Constitution de la liberté ( $1^{\mathrm{er}}$ octobre 1935$)$ et La législation national-socialiste et la réserve de l'ordre public dans le droit international privé (28 novembre 1935). Selon Zarka, ces deux textes montrent non seulement l'adhésion totale de Schmitt à l'égard des trois lois principales de Nuremberg censées renforcer la pureté de l'identité allemande (le drapeau, la citoyenneté et le sang), mais la radicalité avec laquelle Schmitt conçoit par-là la nouvelle (pour ne pas dire l'unique) constitution de la liberté de l'Allemagne. Deux motifs principaux et intrinsèquement liés sont au cœur de cette « nouvelle constitution » proposée par les lois de Nuremberg, selon l'auteur : le rejet de la conception libérale du politique, hors du champ légitime du politique à la faveur de la conception national-socialiste et « la désignation claire de l'ennemi »; celle-ci est corrélative de la mise en évidence des fondements de l'ordre völkisch: le peuple allemand, le mouvement national-socialiste, l'État et l'armée avec le Fürhrer comme chef (p. 22). Cette nouvelle constitution de la liberté pourra donc, au moment opportun, légitimer le nouvel État allemand comme un État d'exception. Cette idée d'État d'exception est très importante dans le décisionnisme schmittien: l'essence pure du politique, ce n'est pas la normalité du quotidien bien ancrée solidement dans ses institutions reconnues avec ses règles toute faites d'avance, comme l'affectionne le libéralisme ; l'essence du politique, c'est la décision pure et le sens de cette décision pure n'est jamais aussi visible que dans l'État d'exception. Pour Schmitt la véritable essence du politique se manifeste dans toute sa pureté dans celui qui décide lors d'une situation exceptionnelle. Cette décision qui se prend hors de toutes normes habituellement reconnues est la seule, selon Schmitt, qui atteint les fondements du droit. La souveraineté absolue s'incarnerait dans la capacité d'un peuple à assumer cette décision pure.

On comprendra ici que cette position non seulement rejette radicalement le libéralisme politique mais recèle un danger énorme, celui d'une possibilité de légitimer ce 
qui «normalement» n'est pas légitimable. C'est exactement le cas de la nature de la lutte raciale entre la race allemande et la race juive que Schmitt, on le verra dans le second texte analysé, vient à légitimer dans les lois de Nuremberg au nom de l'État d'exception et du droit privé international. Autrement dit, ce problème ne concerne que les deux protagonistes et ne vise « qu'à protéger et à défendre ce qui est allemand [...] [L]a défense et la protection du "sang allemand» impliquent de prendre les mesures législatives nécessaires pour éviter la contagion, la dégénérescence, la souillure et le déclin de la race allemande qui trouvent leur cause dans la race juive » (p. 30). Schmitt en vient à légitimer "de plein droit» la sortie hors du droit positif des juifs allemands et même des juifs européens. En ce sens, « la législation raciale de Nuremberg fournit un exemple particulièrement probant, peut-être le seul probant, du concept schmittien de politique»( p. 25). Cette législation ne viendrait, selon Schmitt, que remettre sur pied ce que le libéralisme a occulté : «la distinction de ceux qui appartiennent à la race allemande et de ceux qui n'en relèvent pas » (p. 24). Comme il n'y a pas, dans la logique de Schmitt, de souveraineté sans la désignation d'un ennemi de cette dite souveraineté, en même temps qu'elles viennent fonder une nouvelle constitution de la liberté du peuple allemand, les lois de Nuremberg définissent la nature du nouvel ennemi. Cet ennemi est "celui qui n'est pas de substance allemande ni de sang allemand, si peu que ce soit: le juif », écrit Zarka (p. 25).

Le second chapitre cherche à établir le concept schmittien de l'ennemi « substantiel ». Figure absolue de l'ennemi, le juif, soutient Zarka, apparaît, sans être nommé de la sorte, comme celui qui peut justifier l'extermination totale. Afin de cerner le concept d'ennemi substantiel, Zarka reprend ici la double définition de l'ennemi, définie par Schmitt, de l'inimicus (qui est de nature privée) et de l'hostis (qui est de nature politique), et il la réinterprète selon les catégories " ennemi relationnel » et " ennemi substantiel ». Est de nature relationnelle l'ennemi qui tombe sous les faits de circonstances, il ne s'agit d'une tendance inscrite ni dans la nature humaine en général ni dans celle des États. Si une dynamique de l'hostilité peut s'instaurer, c'est parce que les hommes ou les États se trouvent entraînés dans un désir illimité de puissance qui les oppose, dont la raison est la volonté pour chacun d'assurer sa sécurité propre pour l'avenir » (p. 38). On trouve ici la conception hobbesienne, reprise par Schmitt, sur la légitimité de la guerre, laquelle dépend donc essentiellement des circonstances relationnelles entre puissances antagonistes. Le problème, soutient Zarka, c'est qu'au-delà de cette conception relationnelle de l'ennemi se trouve chez Schmitt une conception très radicale de l'ennemi qui n'est pas fonction de la relation effective mais qui s'inscrit dans la teneur substantielle de l'ennemi. L'ennemi substantiel n'est pas un ennemi de circonstance mais un ennemi "de race et de sang ». Le juif, contrairement à d'autres races, n'est pas assimilable, «il peut se déguiser mais n'est pas assimilable» (p. 39). Le juif est l'ennemi substantiel, car il n'est pas « un ennemi comme les autres, il est celui avec lequel se jouent un affrontement séculaire et une bataille décisive, celle de l'avenir de la civilisation» (p. 42). En me défendant contre le juif, écrivait Schmitt « je lutte pour l'œuvre du Seigneur ». Le concept d'ennemi substantiel apparaîtra donc central dans la position que soutient Zarka contre Schmitt.

On ne peut que déplorer ici le fait que ce chapitre, fort original, ne soit pas plus développé, compte tenu de la charge qu'entreprend Zarka contre la pensée de Schmitt. Sous un autre angle, le dernier chapitre reprend la question de l'ennemi substantiel. S'il a pensé cette "sortie de l'ennemi hors de l'humanité, hors de la valeur, sous la figure de l'ennemi absolu ", Schmitt a pourtant esquivé la désignation de l'ennemi du 
nazisme au profit d'une autre guerre, plus redoutable, celle de la guerre atomique. Schmitt résorberait la question de l'ennemi substantiel en déplaçant la question de l'extermination par «les potentialités ouvertes par la nouvelle arme» (p. 43). Face à un ennemi absolu il faut développer un moyen de destruction absolu, de telle façon que c'est la nature de l'ennemi qui détermine la nature des moyens pour l'exterminer. Mais on n'aura pas chez Schmitt, déplore lui-même Zarka, une définition plus explicite de l'ennemi substantiel. On trouvera cependant cette phrase étonnante de Schmitt: «Les hommes qui utilisent ces moyens contre d'autres se voient contraints d'anéantir aussi moralement ces autres hommes, leurs victimes et leurs objets. Ils sont forcés de déclarer criminel et inhumain dans son ensemble le camp adverse, d'en faire une nonvaleur totale, sous peine d'être eux-mêmes des criminels ou des monstres. La logique de la valeur et de la non-valeur déploie sa pleine rigueur destructrice et contraint à des discriminations. À des criminalisations et à des dépréciations toujours nouvelles, toujours plus profondes, jusqu'à l'extermination de tout sujet sans valeur, indigne de vivre» (p. 44).

Le nazisme, écrit Zarka, a-t-il été autre chose ? Pourtant, s'il en fut ainsi, «ce n'est pas un accident de l'histoire ». Ainsi, loin de rejeter les écrits de Carl Schmitt, Zarka soutient qu'il faut les lire ! Lire les œuvres de Schmitt, car c'est là « ce que l'homme a fait de pire» (p. 49), et c'est pourquoi il faut justement le lire. Mon intention, écrit-il en réponse aux critiques qui lui sont adressées, «n'a jamais été, ce que certains m'ont reproché pourtant, de censurer les textes de Schmitt ou d'empêcher qu'ils soient publiés ou lus. [...] Ma démarche est exactement le contraire ; elle consiste à dire qu'il importe de mettre à la disposition du public tous les textes de Schmitt, y compris ceux de la période nazie et ceux qui prolongent ces positions après le nazisme » (p. 90-91). Il ne s'agit pas non plus de blanchir Schmitt de quoi que ce soit. Position diamétralement opposée à celle d'Emmanuel Faye qui cherchait plutôt à rayer des bibliothèques les écrits de Heidegger, la position de Zarka en est une qui appelle au débat, mais cela sans oublier que "la barbarie nazie, la réalité du génocide a été possible dans l'histoire du $\mathrm{XX}^{\mathrm{e}}$ siècle parce qu'elle avait d'abord été pensée et admise par certains comme possible. La pensée de Schmitt va dans cette direction de l'ignominie » (p. 90). On attendra avec impatience l'autre livre annoncé par Zarka, pour comprendre davantage les fondements de sa propre position contre Schmitt. Cela en méditant longuement, oui, l'ampleur de la séduction qu'a exercé un tel régime sur Schmitt, mais surtout comment cette séduction fait son chemin dans le renouveau du marxisme.

LUC VIGNEAULT

Université de Moncton

Campus d'Edmundston 